\title{
Analysis of effect of Meditation on Cognitive Load using Higher Order Crossing Features
}

\author{
N. Jadhav, R. Manthalkar and Y. Joshi \\ Department of Electronics \& Telecommunication Engg.,SGGSIE\&T, Nanded- India \\ \{jadhavnarendra,rrmanthalkar,yvjoshi\}@sggs.ac.in
}

\begin{abstract}
In this paper, the effect of meditation on the seven load arithmetic tasks using Electroencephalogram (EEG) is investigated. The focused attention on breathing is taught to 11 healthy subjects as a simple meditation technique. EEG signals have been recorded at the beginning of meditation and after four weeks of regular meditation (daily 20 minutes) using EMOTIV device. The higher order crossings (HOC) features are derived from EEG signals for analysis of seven cognitive loads. The pre and post-experiment results are compared based on reaction time and effect on brain lobe. The K-Nearest Neighbors (K-NN) classifier is used for classification of seven loads and achieved $83.57 \%$ accuracy in the post and $89.61 \%$ accuracy in pre-experiment. The EEG-based cognitive load analysis is an important tool for continuous memory load measurement application.
\end{abstract}

Keywords: EEG, EMOTIV, Cognitive Load, Meditation, K-NN

\section{Introduction}

Cognitive load is an indicator of mental effort that is being used in the cognitive task. Cognitive monitoring [1] is a measurement of the load imposed on the working memory to avoid cognitive overload during a critical situation like air traffic control and emergency operations. In student life, the cognitive loads increase due to cognitive demands during examination like memorization and mathematical calculations. In order to reduce the anxiety and fear during critical situations, the physical and mental stability are important. The meditation is an ancient technique considered for body and mind regulation. The meditation plays an important role at physical, spiritual, and mental levels. EEG has an excellent temporal resolution in the millisecond than PET and fMRI. The human thoughts are formed due to the electrical activities of neurons within the brain. These activities are possible to record due to advances in brain sensing technology such as wireless EMOTIV EPOC+ device [2]. The cognitive loads are measured by different methods such as subjective method [3, 4], and behavioral \&physiological measures. Physiological measures consist of heart rate variability (HRV) [7], galvanic skin response (GSR) [8], and EEG [1]. The brain activity recorded using EEG is recognized as perfect cognitive load reflector among these methods. The meditation techniques are classified as focused attention and open monitoring meditation [10]. In this paper, the focused attention meditation is used. Meditation affects many cognitive abilities. The stress and anxiety are reduced by the meditation practice. The four weeks meditation practice is considered in this work. EEG features obtained in pre and post-experiment are compared to investigate the effect of meditation. The objective of this paper is to investigate the effect of meditation on arithmetic task load using HOC features. This experiment has not been studied yet.

\subsection{The Background}

As per literature survey, EEG-based cognitive loads mostly studied cognitive tasks, memory performance, reaction time, and cognitive processes. Zarjam et al. [11,12] investigated memory load using EEG signals during cognitive task inducing seven levels of workload using arithmetic tasks. The $98 \%$ classification accuracy has been achieved using wavelet features like entropy, energy, and standard deviation, across twelve subjects for seven load levels [12].

B. Iyer, S. Nalbalwar and R.Pawade (Eds.)

ICCASP/ICMMD-2016. Advances in Intelligent Systems Research.

Vol. 137, Pp. 433-439.

(C) 2017- The authors. Published by Atlantis Press

This is an open access article under the CC BY-NC license (http://creativecommons.org/licens)es/by-nc/4.) 
In [13-16], the brain lobe variations for different load levels have been studied using EEG signals. It has observed that as load level increases, the variation in different brain lobes increases and the frontal theta activity increases [16]. The effect of mindfulness meditation on cognitive load is discussed in [10, 18, 19]. The significant improvement in attention after 4 weeks of meditation (daily 20 minutes) than the control group is studied in [18]. The cognitive load in pre and post-meditation has assessed using the Stroop task and the d2-test of attention [19]. The clinical and psychological effects of meditation are summarized in [10].

\subsection{The Contribution}

There are three steps to access the enhancement of cognitive ability using meditation as:

1. To assess the cognitive load using EEG signals is known as pre-experiment. The cognitive load inducing seven levels of load using arithmetic tasks.

2. Regular practice of meditation for 20 minutes with awareness for 4 weeks.

3. Repeat step one after 4 weeks. This is known as post-experiment.

After above three steps, the investigations of enhancement of cognitive load results are compared in pre and post-experiment. EEG signals recorded during a cognitive task is correlated with an assessment of memory involves in a task using HOC features and classify the seven load levels using K-NN classifier.

Based on previous studies, the effect of meditation on arithmetic cognitive load based on EEG signals has not been studied yet. The contributions of this work are: To investigate the effect of meditation on the seven load arithmetic tasks using HOC features and classify the load levels using K-NN classifier. Compare pre and post experiment results based on reaction time and effect on brain lobe. The paper is organized as follows: Section2 is discussed about the material and methods. In section 3, the pre-processing, feature extraction and data classification are discussed. The results and discussion are presented in section 4 . The conclusion is discussed in section 5 .

\section{Material and Methods}

\subsection{The subjects}

EEG signals are recorded from 11 healthy (6 males and 5 females) institute students in the age group of 22.32 $( \pm 1.1)$ years participated voluntarily as the subjects in pre and post experiment. Before the experiment, the subjects have signed a consent form and have been introduced about cognitive tasks and meditation instructions. The subjects performed daily meditation of 20 minutes with spine erect and concentrate on breathing with awareness of inhale and exhale.

\subsection{EEG data recording}

EEG signals are recorded using EMOTIV EPOC+ wireless EEG device on a separate recording laptop. The arithmetic tasks are displayed using another laptop, $70 \mathrm{~cm}$ away from the subject in order to minimize eye movements. The EEG recording has been done using fourteen electrodes EMOTIV EPOC+ headset with sampling frequency $128 \mathrm{~Hz}$. The fourteen electrodes:AF3, AF4, F3, F4, FC5, FC6, F7, F8, T7, T8, P7, P8, O1, and O2are based on the international 10-20 EEG format. The EEG is recorded during seven arithmetic task levels in pre and post experiment of the same subject.

The subjects are asked to avoid any physical movements to minimize the artifacts of muscle movement. The correct answer is indicated by showing finger up by keeping a hand in a fixed position. The subject could make eye blinks or movements in 30 seconds' break between each two levels.

\subsection{Experimental protocol}

The protocol of this work is same as used in $[11,12]$. The cognitive load is of seven different addition load levels. The first load level is simple one digit addition without carry and the last level is three digit additions with three carries. The load level displayed from very low (easy) level to extremely high (difficult) level. The task of each level is shown in Table 1.

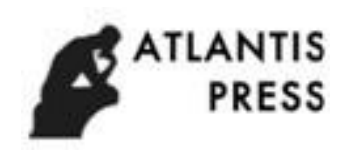


Table 1. Task of each level

\begin{tabular}{clc}
\hline Level & \multicolumn{1}{c}{ Task } & Example \\
\hline L1 (easy) & 1\&2 digit numbers addition & $55+3$ \\
L2 & 1\&2 digit numbers addition with one carry & $64+6$ \\
L3 & 2 digit numbers addition with one carry & $64+62$ \\
L4 & 2 digit numbers addition with two carries & $77+33$ \\
L5 & 2\&3 digit numbers addition with one carry & $288+21$ \\
L6 & 2\&3 digit numbers addition with two carries & $399+34$ \\
L7 (difficulty) & 3 digit numbers addition with three carry & $978+895$ \\
\hline
\end{tabular}

The task starts by displaying start at the center of the screen for 2 seconds so subjects become ready for the task. Each number is displayed at the center of the laptop screen sequentially for 2 seconds. Then the black screen has shown for 1 second which demands on working memory. The memory demands are for arithmetic rules and doing the calculation with or without carry. The multiple-choice answers have been shown for 8 seconds after 1 second retention time. There were six tasks in each level, total 42 additions in seven levels as shown in Fig. 1.

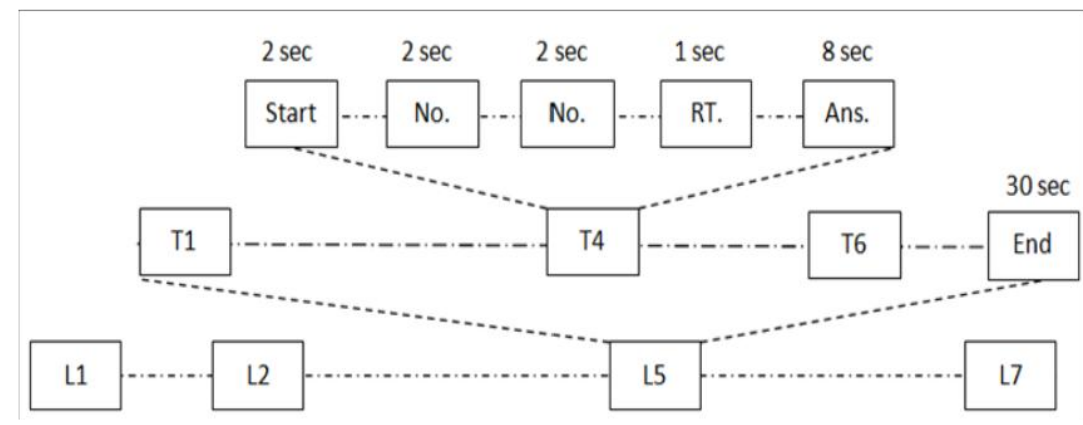

Fig. 1 Protocol of experiment.

No. - Two numbers, RT-Retention time, Ans. - Answer, T1 to T6 - 6 tasks, L1 to L7 - 7 load levels, End - Relax time after each level

After each level, 30 seconds' break is given to subject for relaxation purpose. The time for each level is the same, to impose a cognitive load on the subject when doing addition. EEG signals are recorded for each level in pre and post experiment. Also, reaction time (time required to answer by the subject after displaying multiple choice answers), and performance accuracy (correct answer by subject) of each subject are noted simultaneously. The cognitive load level is increased consistently from easy to difficult level.

\section{Feature extraction and classification}

After EEG recording from each subject, three steps are used: pre-processing, feature extraction and classification which are discussed in this section

\subsection{Pre-processing}

The zero mean EEG data of each subject is pre-processed using band pass filter in 1 to $45 \mathrm{~Hz}$ for removing the artifacts. The band pass filter is designed using FIR filter with Hamming window of length $\mathrm{L}=\mathrm{N}+1$.

The filter length Nis calculated by Kaiser Window method with cut-off frequency 1 to $45 \mathrm{HZ}$ and deviation $[0.05,0.01]$. After removing artifact, the load levels are extracted from the EEG data. The EEG signals are extracted into 90 seconds ( 15 seconds $\mathrm{x}$ 6tasks) epoch corresponding to the duration of each load level. Then the EEG signals of 5 seconds' length are segmented for feature extraction method.

\subsection{Feature Extraction}

The time domain HOC feature is used in this work. The zero mean time series is represented as $X_{t}$, where $t=1$, 2. . $\mathrm{N}$ is up and down oscillation about zero levels and can be used for the zero crossing count. The original os- 
cillations of time series are changed due to filtering; hence zero count also changed. Under this point of view, filter and count procedure could be used to count zero crossing. This process is known as HOC [20].

A high pass filter is given by difference operator $\nabla$ as

$$
\nabla X_{t} \equiv X_{t}-X_{t-1}
$$

A high pass filter sequence is defined as

$$
\nabla^{k-1} X_{t}, k=1,2, \ldots ; t=1,2, \ldots, N
$$

A time series $\mathrm{X}_{\mathrm{t}}(\mathrm{k})$ is constructed for the estimation of the number of zero crossings by symbols 1 (for zero crossings) or 0 (non-crossing) and is given by

$$
X_{t}(k)= \begin{cases}1, & \text { if }\left(\nabla^{k-1} X_{t}\right) \geq 0 \\ 0, & \text { if }\left(\nabla^{k-1} X_{t}\right)<0\end{cases}
$$

The corresponding HOC can be estimated by

$$
D_{k}=N Z C \nabla^{k-1} X_{t}
$$

where, NZC is the number of zero crossing and the desired HOC is then estimated by counting symbol changes in time series $\mathrm{X} 1(\mathrm{k}), \mathrm{X} 2(\mathrm{k}), \ldots ., \mathrm{XN}(\mathrm{k})$, i.e.

$$
D_{k}=\sum_{t=2}^{N}\left[X_{t}(k)-X_{t-1}(k)\right]^{2}
$$

HOC discrimination power diminishes as order $(\mathrm{k})$ increases. HOC is obtained using the corresponding sequence of zero crossing for different $\mathrm{k}$. The feature vector FVHOC $=[\mathrm{D} 1, \mathrm{D} 2 \ldots \mathrm{D} 10]$ is used for classification. In this work, HOC is estimated with a maximum order of 10.

\subsection{Classification}

The K-NN classifier is nonlinear classifier and very simple. They consist in assigning a feature vector to a class according to its nearest neighbors [22]. In this work, Euclidean distance is used to define the nearest neighbors of a feature vector. The K-NN may prove to be efficient with low dimensional feature vectors. The K-NN classifier is used for classification of seven cognitive loads. The performance of classifier is measured by accuracy and is defined as

$$
\text { Accuracy }=\frac{(T P+T N)}{(T P+T N+F P+F N)}
$$

where, TN is true negative, TP is true positive, FN is false negative and FP is false positive.

The classification analysis result has been verified by 10 -fold cross-validation method to the dataset. The whole EEG dataset is divided into ten subsets. The K-NN is trained with nine subsets and the remaining subset is used for testing. This method has been repeated ten times with each subset being used for testing. After validation, the accuracy was calculated by averaging the performance of each feature type.

\section{Result and discussion}

In this section, we investigate some answers for: What is the effect of meditation on frontal, temporal, parietal, and occipital lobe in pre and post experiment? What is the reaction time in pre and post? What is the effect of meditation on average classification rate?

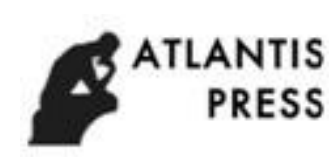




\subsection{HOC for each brain lobe in pre and post experiment}

The HOC rate for each lobe across 11 subjects in pre and post experiment is shown in Fig. 2. The HOC in postexperiment is decreased for all lobes and for all levels except L4 level. This result shows the zero crossing in post-experiment is less i.e. EEG signal variation is less. The HOC in frontal and temporal lobe is more as shown in Fig. 2. The frontal lobe is responsible for short memory and attention and the temporal lobe is responsible for long-term memory. In the seven arithmetic loads, the short and long term memory is involved during tasks [11, 12]. The quantitative analysis of HOC feature for each lobe across all subjects is discussed below.
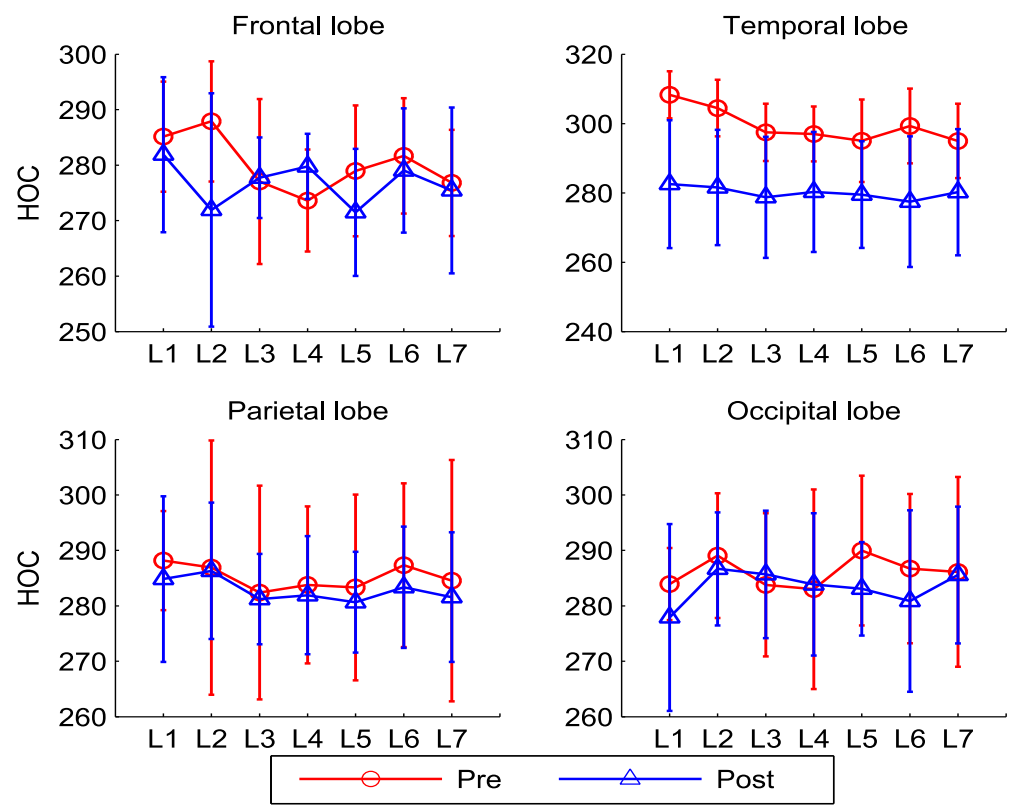

Fig.2 HOC for seven load levels across 11 subjects for each brain lobe in pre and post experiment.

For frontal lobe, the mean (standard deviation) values of HOC feature for seven load levels in pre-experiment are [L1: $285.14( \pm 9.9)$; L2: 287.88 $( \pm 10.8)$; L3: 277.04( \pm 14.8$)$; L4: 273.61 $( \pm 9.1)$; L5: 278.97 $( \pm 11.7)$; L6: 281.68( \pm 10.3$)$; L7: $276.80( \pm 9.5)]$ and in post-meditation, the mean (standard deviation) values are [L1: 281.88 $( \pm 13.9)$; L2: $271.91( \pm 21)$; L3: $277.03( \pm 7.2)$; L4: $279.76( \pm 5.8)$; L5: $271.48( \pm 11.4)$; L6: $279.03( \pm 11.2)$; L7: $275.44( \pm 14.9)]$. The statistical values of the HOC are less in post-experiment (except L4) than in preexperiment which shows that zero crossing is decreased in post-meditation.

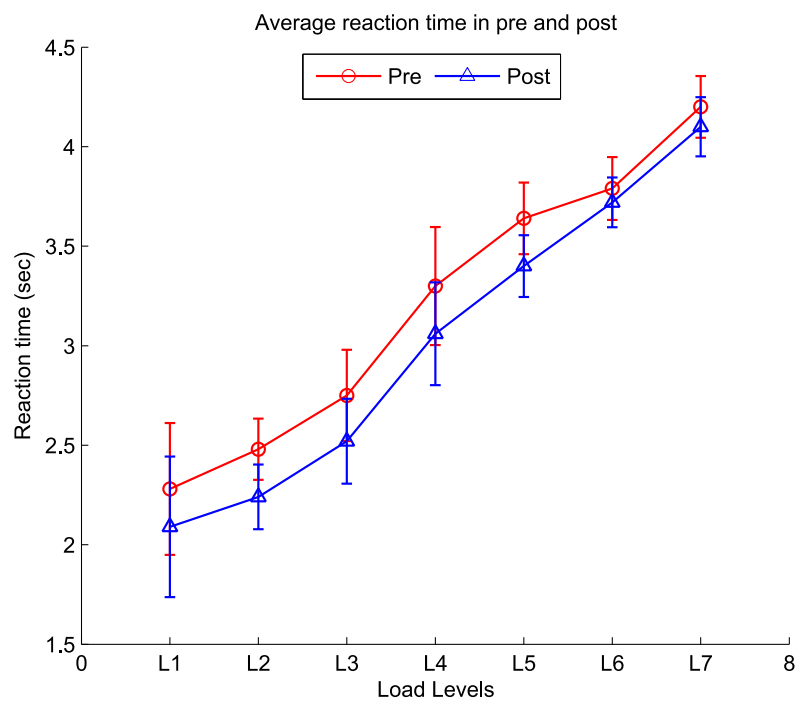

Fig. 3 Average reaction time in pre and post experiment for seven load levels 
The mean (standard deviation) values of HOC feature for seven load levels in pre-experiment for temporal are [L1: $308.28( \pm 6.7)$; L2: 304.46 ( \pm 8.1$)$; L3: 297.45 ( \pm 8.2$)$; L4: 296.99 ( \pm 7.9$)$; L5: 295.01 ( \pm 11.8$)$; L6: 299.30 $( \pm 10.7)$; L7: $294.97( \pm 10.7)]$ more than in post-meditation [L1: $282.52( \pm 18.4)$; L2: $281.55( \pm 16.6)$; L3: 278.72 ( \pm 17.4$)$; L4: 280.27 ( \pm 17.2$)$; L5: $279.50( \pm 15.4)$; L6: $277.48( \pm 18.8)$; L7: $280.19( \pm 18.1)]$. The statistical values of the HOC show that the effect of meditation on temporal lobe is decreased in post-meditation. The variation of HOC for parietal and occipital lobe is less in pre and post-experiment.

\subsection{Average reaction time in pre and post experiment}

The reaction time in post-experiment is decreased than the pre-experiment as shown in Fig. 3. As load level increases, the reaction time increases in pre and post experiment. This result indicates that the load on memory increases as task level increases. The reaction time is measured by putting the marker when subject answers after displaying multiple choices. The reaction time for the post-experiment and pre-experiment is $3.01( \pm 0.07)$ and $3.20( \pm 0.06)$ across all levels respectively. The reaction time in post-experiment is less than the pre-experiment by $6 \%$.

\subsection{Classification accuracy}

The average classification accuracy of K-NN classifier using HOC features is shown in Fig. 4. The classification accuracy decreases as load level increases in pre and post-experiment. The maximum classification accuracy is $89.61 \%$ in pre-experiment and $83.57 \%$ in post-experiment for two load levels and minimum classification accuracy $64.75 \%$ in pre-experiment and $58.16 \%$ in post-experiment for seven load levels. The accuracy in the post-experiment is decreased indicates the equanimity achieved in the subjects after 4 weeks of meditation. The equanimity represents the calmness in the subjects in post-experiment.

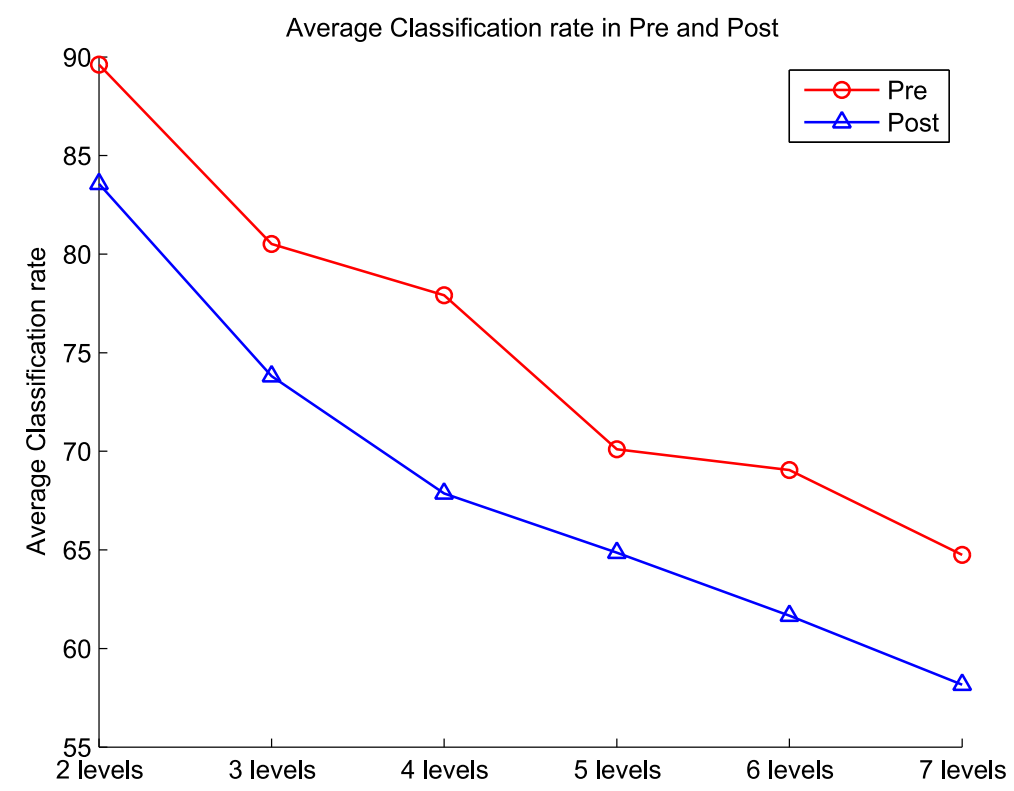

Fig. 4 Average accuracy rate in pre and post experiment for each level

\section{Conclusions}

A new HOC based classification method has been proposed here for investigation of the effect of meditation on seven level cognitive loads. This method has exposed the potential of cognitive load measurement method from EEG signals. The highest average classification accuracy of K-NN is $89.61 \%$ obtained for cognitive load. The effect of the cognitive load has been observed at the frontal and temporal lobe. The reaction time has been increased as load level increases. In future, the reaction time could be monitored by video recording to avoid the human error and improve classification accuracy by using different classifiers. These would be used in the education system for improving the engagement of students in learning.

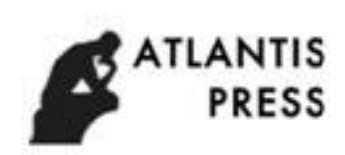




\section{References}

[1] Pavlo Antonenko, Fred Paas, Roland Grabner, and Tamara van Gog. Using electroencephalography to measure cognitive load. Educational Psychology Review, 22(4):425-438, 2010.

[2] Emotiv Epoch Headset. Available at: http://www.emotiv.com. Accessed on: 31st August, 2013.

[3] Fred GWC Paas, Jeroen JG Van Merriënboer, and Jos J Adam. Measurement of cognitive load in instructional research. Perceptual and motor skills, 79(1):419-430, 1994.

[4] Najmedin Meshkati, Peter A Hancock, Mansour Rahimi, and Suzanne MDawes. Techniques in mental workload assessment. 1995.

[5] Bo Yin and Fang Chen. Towards automatic cognitive load measurementfrom speech analysis. In International Conference on Human-Computer Interaction, pages 1011-1020. Springer, 2007.

[6] Natalie Ruiz, Ronnie Taib, Yu David Shi, Eric Choi, and Fang Chen. Usingpen input features as indices of cognitive load. In Proceedings of the 9th international conference on Multimodal interfaces, pages 315318. ACM, 2007.

[7] Fred GWC Paas and Jeroen JG Van Merriënboer. Instructional controlof cognitive load in the training of complex cognitive tasks. Educational psychology review, 6(4):351-371, 1994.

[8] Yu Shi, Natalie Ruiz, Ronnie Taib, Eric Choi, and Fang Chen. Galvanicskin response (GSR) as an index of cognitive load. In CHI'07 extended abstracts on Human factors in computing systems, pages 2651-2656. ACM, 2007.

[9] Thorsten O Zander and Christian Kothe. Towards passive brain-computerinterfaces: applying braincomputer interface technology to human- machine systems in general. Journal of neural engineering, 8(2):025005, 2011.

[10]B Rael Cahn and John Polich. Meditation states and traits: EEG, ERP, andneuroimaging studies. Psychological bulletin, 132(2):180, 2006.

[11]Pega Zarjam, Julien Epps, Fang Chen, and Nigel H Lovell. Estimatingcognitive workload using wavelet entropy-based features during an arithmetic task. Computers in biology and medicine, 43(12):2186-2195, 2013.

[12]Pega Zarjam, Julien Epps, and Nigel H Lovell. Beyond subjective self-rating: EEG signal classification of cognitive workload. IEEE Transactions on Autonomous Mental Development, 7(4):301-310, 2015.

[13]Pega Zarjam, Julien Epps, Nigel H Lovell, and Fang Chen. Characterization of memory load in an arithmetic task using non-linear analysis of EEG signals. In 2012 Annual International Conference of the IEEE Engineering in Medicine and Biology Society, pages 3519-3522. IEEE, 2012.

[14] Markus Näpflin, Marc Wildi, and Johannes Sarnthein. Test-retest reliability of EEG spectra during a working memory task. Neuroimage, 43(4):687- 693, 2008.

[15]Joshua Jacobs, Grace Hwang, Tim Curran, and Michael J Kahana. EEG oscillations and recognition memory: theta correlates of memory retrieval and decision making. Neuroimage, 32(2):978-987, 2006.

[16]Ole Jensen and Claudia D Tesche. Frontal theta activity in humans increases with memory load in a working memory task. European journal of Neuroscience, 15(8):1395-1399, 2002.

[17]B Schack, W Klimesch, and P Sauseng. Phase synchronization betweentheta and upper alpha oscillations in a working memory task. International Journal of Psychophysiology, 57(2):105-114, 2005.

[18]Fadel Zeidan, Susan K Johnson, Bruce J Diamond, Zhanna David, andPaula Goolkasian. Mindfulness meditation improves cognition: Evidence of brief mental training. Consciousness and cognition, 19(2):597-605, 2010.

[19]Adam Moore and Peter Malinowski. Meditation, mindfulness and cognitive flexibility. Consciousness and cognition, 18(1):176-186, 2009.

[20]Benjamin Kedem and Sidney Yakowitz. Time series analysis by higher order crossings. IEEE press New York, 1994.

[21] Panagiotis C Petrantonakis and Leontios J Hadjileontiadis. Emotion recognition from EEG using higher order crossings. IEEE Transactions on Information Technology in Biomedicine, 14(2):186-197, 2010.

[22] Richard O Duda, Peter E Hart, and David G Stork. Pattern classification.John Wiley \& Sons, 2012. 